\section{BMJ Open Respiratory Research}

\title{
Visual rating assessment of cerebral atrophy and its relationship with cognitive function in chronic obstructive pulmonary disease
}

\author{
Charlotte Claire Savage, ${ }^{1}$ Piers Henry Anderson Dixey, ${ }^{2}$ Catherine Pennington, ${ }^{3}$ \\ James W Dodd ${ }^{2}$
}

To cite: Savage CC, Dixey PHA, Pennington $\mathrm{C}$, et al. Visual rating assessment of cerebral atrophy and its relationship with cognitive function in chronic obstructive pulmonary disease. BMJ Open Resp Res 2018;5:e000310. doi:10.1136/ bmjresp-2018-000310

- Additional material is published online only. To view please visit the journal online (http://dx.doi.org/10.1136/ bmjresp-2018-000310).

Received 16 May 2018 Revised 23 September 2018 Accepted 24 October 2018

Check for updates

(C) Author(s) (or their employer(s)) 2018. Re-use permitted under CC BY-NC. No commercial re-use. See rights and permissions. Published by BMJ.

${ }^{1}$ Faculty of Health Sciences, University of Bristol, Bristol, UK

${ }^{2}$ Academic Respiratory Unit, Clinical Sciences, University of Bristol, Bristol, UK

${ }^{3}$ Neurology, School of Clinical Sciences, University of Bristol, Bristol, UK

Correspondence to Dr James W Dodd; james.dodd@bristol.ac.uk; james.dodd@nbt.nhs.uk

\section{ABSTRACT}

Introduction Widespread white matter damage and cognitive impairment have been demonstrated in chronic obstructive pulmonary disease (COPD). However, it remains unclear if brain atrophy is a global phenomenon or if specific subregions are differentially affected. The aims of this study are, first, to test a simple, validated visual analogue grading technique. Second, we hypothesised that frontal regions of the brains of patients with COPD will show greater signs of atrophy compared with control subjects. Third, any localised regions of atrophy would correlate with components of cognitive performance. Finally, the severity of cerebral atrophy would be associated with measures of respiratory disease severity. Methods We used a simple, validated visual analogue grading technique to assess the degree of regional atrophy in multiple brain regions from cerebral MR images in patients with stable non-hypoxaemic COPD $(n=25)$ and age-matched control subjects $(n=25)$. We also explored correlations between regional brain atrophy with demographics, cognitive performance measures and disease severity. Measures of cognitive performance focused on executive function, working memory, verbal memory, overall memory and processing speed. Measures of disease severity include lung function, gas exchange, health status and breathlessness questionnaires.

Results The visual grading scale found that patients with COPD had significantly greater frontal atrophy than control subjects $(p=0.02)$, independent of smoking history, comorbid depression or anxiety. Cognitive function was significantly worse in the COPD group for executive function $(p<0.001)$, working memory $(p=0.02)$, verbal memory $(p=0.03)$ and processing speed $(p=0.001)$. Group differences in atrophy did not appear to account for differences in cognitive function. We were unable to identify meaningful correlations between regional atrophy and disease severity or cognitive function.

Conclusion Further work is needed to identify causative mechanisms behind unexplained structural brain changes in COPD.

\section{INTRODUCTION}

It is well established that patients with chronic obstructive pulmonary disease (COPD) have multiple comorbidities, such as heart

\section{Key messages}

Significant brain pathology and cognitive impairment has been consistently demonstrated patients with chronic obstructive pulmonary disease (COPD), but it is not clear how best to detect and measure these brain changes. Current MR analysis requires complex data processing and expertise to interpret

- A simple, easy to learn visual rating scale may be able to detect significant brain pathology in COPD.

> We used a simple, validated visual analogue grading technique to assess the degree of regional atrophy in multiple brain regions from cerebral MR images in patients with COPD. It was simple to learn and repeatable and shows patients with COPD had significantly greater frontal atrophy than control subjects.

disease, stroke, skeletal muscle wasting and depression. $^{12}$ These systemic effects are not explained by shared risk factors alone, such as cigarette smoking, hypertension of hypoxaemia, leading to the hypothesis of a 'COPD-specific' risk for comorbidity. ${ }^{3}$ The presence of comorbidities in COPD is associated with significantly worse patient outcomes such as increased hospitalisations, impaired functional capacity, decreased quality of life and mortality. ${ }^{3}$ Therefore, characterising and understanding these comorbidities is essential to optimising the management of each individual patient.

Previous studies have shown that up to $77 \%$ of patients with hypoxaemia with COPD have cognitive impairment indicative of cerebral dysfunction. ${ }^{4}$ This has also been demonstrated in non-hypoxaemic patients with $\mathrm{COPD}{ }^{5}$ There is also evidence that cognitive impairment in COPD is associated with reduced ability to carry out basic and instrumental activities of daily living ${ }^{67}$ and poor medication adherence. ${ }^{8}$ Combined, these factors are likely to contribute to increased exacerbations and hospital admissions, decreased quality of life, 
increased length of hospital stay ${ }^{9}$ and, possibly, increased mortality. ${ }^{10}$ The identification of COPD as a risk factor for accelerated cognitive dysfunction ${ }^{11}$ presents clinicians and researchers with a potential target for interventions to prevent or delay cognitive impairment.

People with COPD have been found to perform poorly across a wide spectrum of cognitive areas. The most frequent and severely affected cognitive domains appear to be those related to executive function, attention and memory. ${ }^{5}$ Motor function and processing speed are also likely to be negatively impacted on by COPD. ${ }^{12}$ We may therefore expect specific regions of the brain, thought to be functionally linked to these cognitive domains, to be at risk of damage in patients with COPD, for example, executive function and frontal lobes.

Previous analysis of global microstructural changes in this population of stable, non-hypoxaemic COPD MR diffusion tensor imaging showed extensive reduction in white matter integrity, but no difference in the overall volume of grey or white matter, suggesting generalised cerebral atrophy, was unlikely. ${ }^{13}$ Two small studies in patients with hypoxaemia with COPD found evidence of frontal lobe hypoperfusion with the authors concluding that anterior cerebral hypoperfusion could herald frontal-type cognitive decline with the worsening of the hypoxaemia. ${ }^{1415}$ Hippocampal atrophy has also been identified in $\mathrm{COPD}^{16}$ in addition to localised grey matter (GM) loss. ${ }^{17} 18$ However, these studies are limited, as they require complex data processing and statistical techniques, which do not always adequately adjust for multiple comparisons.

In this study, we test a simple, validated visual MRI rating scale to analyse multiple brain regions of stable, non-hypoxaemic patients with COPD and age-matched control subjects. We aimed to identify areas of regional brain atrophy, we sought associations between regional brain atrophy and cognitive function before looking at whether any patient characteristics could account for particular regions of atrophy.

There is ongoing debate concerning the neuronal pathways and supplementary cortical and subcortical brain regions involved in cognitive functioning. We used associations more widely accepted to form our hypotheses. The relationship between executive function and the frontal lobe ${ }^{19}$ and that between memory and the hippocampus ${ }^{20}$ are examples. We aimed to test a simple, validated visual analogue grading technique in this study. We hypothesised that, first, the frontal regions of the brains of patients with COPD will show greater signs of atrophy compared with control subjects. Second, any localised regions of atrophy would correlate with components of cognitive performance. Finally, the severity of cerebral atrophy would be associated with measures of respiratory disease severity.

\section{METHODS}

\section{Subjects}

This study was carried out as analysis of data collected from a prospective case-control study, titled 'Brain structure and function in chronic obstructive pulmonary disease- $\mathrm{a}$ multimodal cranial magnetic resonance imaging study'. ${ }^{13}$ Twenty-five patients with stable, non-hypoxaemic COPD $(n=25)$ were recruited from outpatient respiratory clinics of two university teaching hospitals (St George's Hospital and Royal Brompton Hospital) between 2010 and 2011. Patients were non-hypoxaemic $\left(\mathrm{PaO}_{2}>7.3 \mathrm{kPa}\right)^{21}$ and had no exacerbations in the preceding 8 weeks. Recruitment of an age-matched control group $(n=25)$ from the local community took place through local press advertisements. All subjects were required to have at least primary school education or equivalent and be competent in English in order to complete neuropsychological testing. Control subjects with significant respiratory or cognitive difficulties were excluded. Exclusion criteria were otherwise the same for both groups and included other causes of cognitive impairment (overt psychiatric illnesses, drugs known to cause sedation or cognitive impairment, diagnosis of dementia, current or past alcohol or drug abuse), breathlessness attributed to heart disease or left ventricular dysfunction, neurologic disease, active tumours, vascular complications of diabetes, diagnosis of obstructive sleep apnoea, visual and hearing disturbances and hepatic failure.

\section{Image acquisition}

Whole-brain T1-weighted MRI was performed on all patients and controls using a Philips 3T Achieva dual TX scanner (with a 32-channel head coil).

\section{Image analysis \\ Technique}

The T1-weighted MRI images were analysed in the coronal plane by a rater blinded to the clinical condition of each subject. A validated visual analogue MRI grading technique was used to assess the degree of atrophy in multiple brain regions. ${ }^{22}$ Fifteen brain regions were rated bilaterally using a 5-point Likerttype scale: $0=$ no atrophy; $1=$ borderline appearances, not necessarily abnormal; $2=$ definitive signs of atrophy; $3=$ severe atrophy, with preservation of structure to a degree; 4=very severe atrophy, GM not discernible in cortical structures. Each structure was located within one of four slices, chosen using defined landmarks (from anterior to posterior), namely: slice $1=\mathrm{at}$ the level where the internal capsule can first be seen; slice $2=$ at the level of the intralimbic gyrus' medial opening; slice $3=$ at the level of the lateral geniculate nucleus; slice $4=$ the level where the fornix can be seen in continuity beside the pulvinar nucleus. In slice 1, the anterior temporal lobe, basal ganglia, orbitofrontal cortex, dorsolateral frontal cortex and anterior cingulate were rated; in slice 2 , the anterior hippocampus, entorhinal cortex, perirhinal cortex, anterior fusiform gyrus, lateral temporal lobe and insula; in slice 3 , the mid-hippocampus and superior temporal gyrus; in slice 

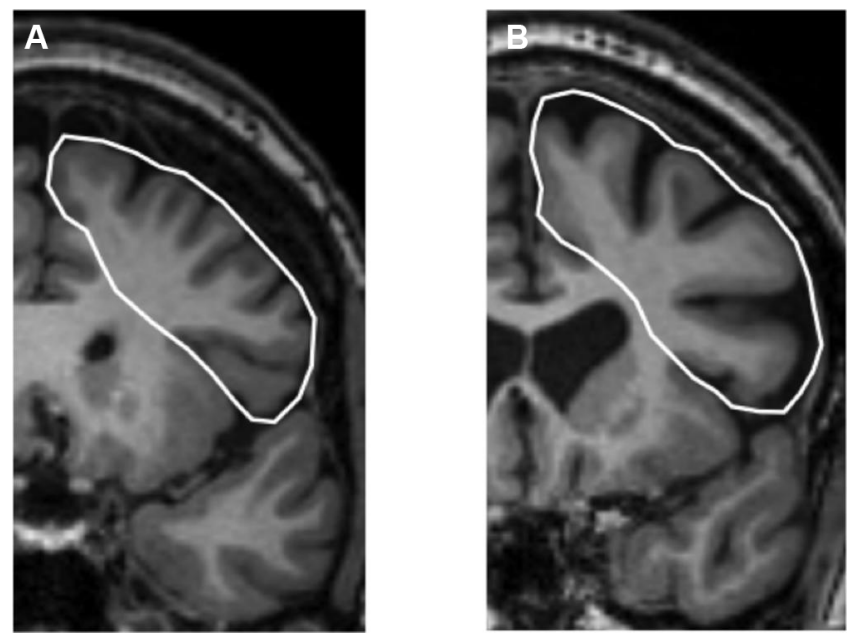

Figure 1 Slice 1 in the right hemispheres of two different subject brains. The white line highlights the dorsalfrontal lobe. This region would be rated as 0 for brain A (not atrophic) and 2 for brain B (signs of atrophy).

4, the posterior hippocampus and Brodmann area 37. Figures 1 and 2 demonstrate rating examples in slice 1 and slice 2 for different subject brains.

\section{Rater training}

The rater (CCS) was trained using a subset of the MRI scans collected for this study, alongside images provided in the original paper describing the technique. The same scan set was independently rated by an experienced rater (CP). An iterative training process was used initially, followed by rerating of 10 scans to give a measure of intrarater reliability (measured using Cohen's kappa coefficient).
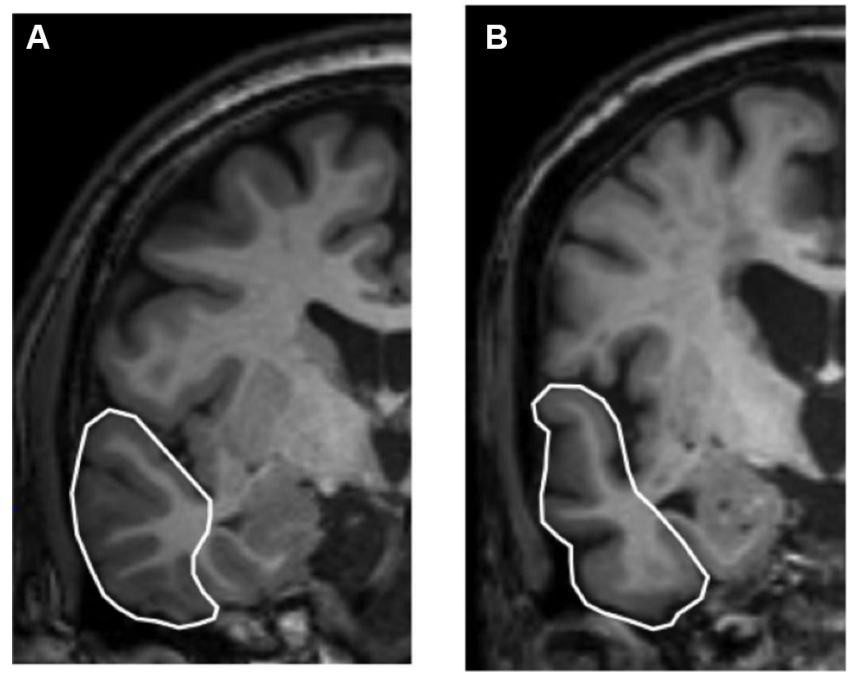

Figure 2 Slice 2 in the left hemispheres of two different subject brains. The white line highlights the lateral temporal lobe. This region would be rated as 0 for brain $A$ (not atrophic) and 2 for brain B (signs of atrophy).

\section{Health assessments}

All participants' demographic and medical histories were collected through interview and medical records. Age, gender, educational level (based on General Certificate of Secondary Education, A level, diploma, degree or other levels of qualification) and pack-years were recorded. The following areas were also explored:

- Comorbidity: Charlson Comorbidity Index (CCI). ${ }^{23}$

- Cerebrovascular risk: modified Framingham's Stroke Risk Profile (FSRP) (ECG-defined left ventricular hypertrophy omitted) ${ }^{24}$

- Mood: the Hospital Anxiety and Depression (HAD) questionnaire. $^{25}$

With regard to the above three scoring methods, the higher the number obtained, the worse outcome predicted.

Additionally, the following were captured for the COPD group only, to define functionality and disease severity:

- Forced expiratory volume in $1 \mathrm{~s}\left(\mathrm{FEV}_{1}\right)$.

- Forced vital capacity.

- $\mathrm{PaO}_{2}$ and $\mathrm{PaCO}_{2}$ using arterialised earlobe capillary blood gases (COPD subjects only).

- St George's Respiratory Questionnaire (SGRQ) - a 50-item questionnaire. A score of 100 indicates the worst possible health status and 0 indicates the best possible health status.

- Medical Research Council Dyspnoea Scale was used to measure patients' perceived respiratory disability, based on their degree of breathlessness.

\section{Neuropsychological tests}

In addition to the Mini-Mental State Examination (MMSE) cognitive screening tool, ${ }^{26}$ a comprehensive neuropsychological battery was used. Executive function was tested using verbal fluency and trail making from the Delis-Kaplan system ${ }^{27}$; working memory using letter-number sequencing and spatial span from Wechsler Adult Intelligence Scale ${ }^{28}$; visual memory using Rey Complex ${ }^{29}$ Figure-Immediate $(3 \mathrm{~min})$ and Delayed Recall (30 min) and Recognition; verbal memory using Word Lists-List Learning, Word Lists Delayed Recall (30 min) and recognition from Wechsler Memory Scale, ${ }^{30}$ and processing speed using the digit symbol and symbol search from Wechsler Adult Intelligence Scale. Testing was carried out in a quiet room to ensure data collection uniformity and in a fixed sequence to avoid responses to self-complete questionnaires being influenced by subsequent tests. Age-standardised scores were then calculated with reference to normative population samples.

Age-standardised cognitive scores were generated with reference to normative population samples. ${ }^{27-30}$ These scores are in the range (mean \pm SD) of $1-20(10 \pm 3)$ for all measures. A score $\leq 7$ indicates mild cognitive impairment. $^{31}$ 


\section{Statistical analysis plan}

Data were analysed using IBM SPSS version 23. Histograms were plotted for each variable to visually check for normality of distribution. Data for demographic characteristics, baseline cognitive function and regional atrophy were assessed for group differences using an independent samples t-test, if normally distributed, or Pearson's $\chi^{2}$ test, if non-normally distributed. Differences were deemed significant at $p<0.05$. Group differences in regional atrophy were also analysed after controlling for CCI, pack-years and HAD scores, using a generalised linear model multivariate analysis. A bivariate correlation was performed in the whole group (COPD and controls) and COPD group to test for associations between regional atrophy and cognitive function (Pearson's correlation coefficient for normally distributed data and Spearman's rank-order correlation for non-normally distributed data). As an exploratory analysis, associations between disease severity and regional atrophy were also assessed in this way.
RESULTS

\section{Demographics}

Demographic characteristics for the control and COPD groups are displayed in table 1 . The groups were well matched on age, sex, FSRP and MMSE score. As anticipated, COPD subjects had a significantly greater number of pack-years (4.16 vs 58.33), they were also significantly more anxious, depressed and with lower levels of education and a greater number of comorbidities than controls.

\section{Visual rating assessment \\ Agreement analyses.}

\section{Inter-rater}

The weighted kappa coefficient at the start of the training process was $0.66(\mathrm{p}<0.001)$, demonstrating significant agreement between the two raters and comparing well to the overall inter-rater kappa value of $0.71 \quad(\mathrm{p}<0.05)$ reported in the paper in which this scale was developed. ${ }^{22}$

\begin{tabular}{|c|c|c|c|}
\hline Characteristic & Control subjects $(n=25)$ & Patients with COPD $(n=25)$ & P values \\
\hline Age (years) & $65.32 \pm 7.85$ & $67.84 \pm 8.16$ & 0.27 \\
\hline Gender (\% female) & 58.30 & 41.70 & 0.40 \\
\hline \multicolumn{4}{|c|}{ Education (\% of each group) } \\
\hline None & 16 & 52 & $0.03^{*}$ \\
\hline Other & 0 & 8 & \\
\hline GCSE & 32 & 20 & \\
\hline A level & 20 & 8 & \\
\hline Diploma & 8 & 8 & \\
\hline Degree & 24 & 4 & \\
\hline Cigarette pack-years & $4.16 \pm 9.71$ & $58.33 \pm 23.80$ & $<0.001^{\star}$ \\
\hline \multicolumn{4}{|c|}{ Charlson Comorbidity Index } \\
\hline 0 & 23 & 0 & $<0.001^{*}$ \\
\hline 1 & 2 & 15 & \\
\hline 2 & 0 & 6 & \\
\hline$\geq 3$ & 0 & 4 & \\
\hline MMSE & $29.28 \pm 0.84$ & $28.08 \pm 1.66$ & 0.07 \\
\hline FSRP & $6.08 \pm 3.13$ & $6.96 \pm 4.02$ & 0.39 \\
\hline HAD scale & $6.76 \pm 5.00$ & $12.05 \pm 8.16$ & $0.009^{\star}$ \\
\hline Executive function & $12.29 \pm 2.57$ & $9.63 \pm 2.21$ & $<0.001^{*}$ \\
\hline Working memory & $11.02 \pm 2.48$ & $9.38 \pm 2.28$ & $0.02^{*}$ \\
\hline Processing speed & $11.54 \pm 2.24$ & $9.22 \pm 2.55$ & $0.001^{\star}$ \\
\hline Memory (all) & $10.93 \pm 3.00$ & $9.33 \pm 2.71$ & 0.05 \\
\hline Visual memory & $10.74 \pm 4.57$ & $9.12 \pm 4.72$ & 0.22 \\
\hline Verbal memory & $11.12 \pm 2.74$ & $9.54 \pm 2.06$ & $0.03^{*}$ \\
\hline
\end{tabular}

Data are presented as mean \pm SD except where indicated otherwise. P values were obtained using independent samples t-test/Pearson's $\chi^{2}$ test depending on normality of distribution.

*Statistically significant result.

COPD, chronic obstructive pulmonary disease; FSRP, Framingham's Stroke Risk Profile; GCSE, General Certificate of Secondary Education; HAD, Hospital Anxiety and Depression; MMSE, Mini-Mental State Examination. 
Table 2 Bilaterally summed composite scores for regional atrophy in the control and COPD groups (presented as mean \pm SD)

\begin{tabular}{lllll}
\hline & $\begin{array}{l}\text { Control subjects } \\
(\mathbf{n}=\mathbf{2 5})\end{array}$ & $\begin{array}{l}\text { Patients with COPD } \\
(\mathbf{n}=\mathbf{2 5})\end{array}$ & P values & Corrected $\mathbf{p}$ values* \\
\hline Frontal & $5.08 \pm 2.68$ & $7.32 \pm 3.26$ & $0.01 \dagger$ & $0.02 \dagger$ \\
\hline Temporal & $5.76 \pm 3.27$ & $7.72 \pm 4.77$ & 0.10 & 0.06 \\
\hline Hippocampal & $4.60 \pm 1.89$ & $5.24 \pm 3.06$ & 0.38 & 0.05 \\
\hline
\end{tabular}

*Generalised linear model multivariate analysis controlling for group differences in Charlson Comorbidity Index, pack-years and Hospital Anxiety and Depression (HAD) scores.

†Statistically significant result.

COPD, chronic obstructive pulmonary disease.

\section{Intrarater}

From the ten rerated scans, 300 values were obtained. Raw percentage agreement was $77.7 \%$ and Cohen's kappa coefficient was $0.66(\mathrm{p}<0.001)$. Again, this compares well to the reported intrarater kappa coefficient of 0.75 $(\mathrm{p}<0.05)$ from the original rating scale paper, indicating good intrarater agreement.

\section{Regional atrophy}

Table 2 shows the bilaterally summed composite scores for regional atrophy in the two groups. Grouping the scores from anatomically and functionally related regions formed the composite scores, as demonstrated in table 3 . Left and right hemisphere scores were summed to give overall bilateral scores and the mean of this value was generated. As per a priori hypothesis, frontal regions of the patients with COPD were more atrophic than those of the control group $(p=0.01)$. This difference in frontal atrophy remained significant after controlling for CCI, pack-years and HAD scores. There was, however, no difference in temporal or hippocampal atrophy between the two groups. After statistical correction, hippocampal atrophy did not meet significance.

\section{Cognitive function}

Neuropsychological test scores significantly worse in the COPD group compared with controls for executive function $(p<0.001)$, working memory $(p=0.02)$, processing

\begin{tabular}{|c|c|}
\hline Group & Regions \\
\hline Frontal & $\begin{array}{l}\text { Dorsolateral frontal cortex } \\
\text { Orbitofrontal cortex } \\
\text { Cingulum }\end{array}$ \\
\hline Hippocampal & $\begin{array}{l}\text { Anterior hippocampus } \\
\text { Mid-hippocampus } \\
\text { Posterior hippocampus }\end{array}$ \\
\hline Temporal & $\begin{array}{l}\text { Anterior temporal lobe } \\
\text { Superior temporal gyrus } \\
\text { Brodmann area } 37 \\
\text { Lateral temporal lobe }\end{array}$ \\
\hline
\end{tabular}

speed $(\mathrm{p}<0.001)$ and verbal memory $(\mathrm{p}=0.03)$, but mean scores for both groups were not in the clinically impaired range (table 1). There was no significant difference in visual memory $(\mathrm{p}=0.22)$ between the two groups. Overall difference in memory function (average of visual, verbal and working memory performance) did not reach statistical significance $(\mathrm{p}=0.05)$.

See online supplementary materials for further description of neuropsychological measures (online supplementary table 1 and online supplementary figures $1-5$ )

\section{Disease severity within the COPD group}

Measures of disease severity for the patients with COPD (table 4) demonstrated no significant hypoxaemia or hypercapnia. The mean age was 67.8 years and $41 \%$ were female. Health status was moderately impaired (SGRQ $51.65)$. Airway obstruction $\left(\mathrm{FEV}_{1}\right)$ was moderate $(52.51 \%$ of predicted value).

\section{Correlations between regional atrophy and cognitive function} Scores for regional atrophy did not significantly correlate with cognitive function in the COPD group. There was a large amount of scatter around the regression line when the association between frontal atrophy and executive function was plotted for the COPD and control groups. On exploration of the whole group (COPD group and control group together) associations between verbal memory and atrophy within the temporal $(\mathrm{r}=0.30$, $\mathrm{p}=0.03)$ and hippocampal $(\mathrm{r}=-0.33, \mathrm{p}=0.02)$ regions were found. However, after correction for multiple comparisons using Bonferroni method $(\mathrm{p}=0.002)$, these associations were not significant.

\section{Disease severity and regional atrophy}

No correlations between regional atrophy in the COPD group and selected measures of disease severity within this group $\left(\mathrm{PaO}_{2}, \mathrm{FEV}_{1} \%\right.$ predicted and SGRQ) were found.

\section{DISCUSSION}

This study set out to use a simple visual MR rating scale ${ }^{22}$ in the assessment of regional cerebral atrophy in patients with COPD and age-matched control subjects. The visual 


\begin{tabular}{|c|c|c|c|}
\hline & Mean \pm SD & Median & IQR \\
\hline $\mathrm{FEV}_{1} \%$ predicted $(\mathrm{n}=23)$ & $52.51 \pm 20.96$ & 48.00 & 31.60 \\
\hline $6 m w d(m)(n=14)$ & $423.25 \pm 55.41$ & 432.00 & 73.13 \\
\hline $\mathrm{PaO}_{2}(\mathrm{kPa})(\mathrm{n}=24)$ & $10.72 \pm 2.07$ & 10.60 & 2.15 \\
\hline $\mathrm{PaCO}_{2}(\mathrm{kPa})(\mathrm{n}=24)$ & $5.09 \pm 0.47$ & 5.01 & 0.67 \\
\hline MRC dyspnoea score ${ }^{n p}(n=21)$ & - & 2 & 1 \\
\hline SGRQ (n=22) & $51.65 \pm 19.98$ & 49.66 & 30.33 \\
\hline
\end{tabular}

6mwd, 6 min walking distance; COPD, chronic obstructive pulmonary disease; FEV ${ }_{1}$, forced expiratory volume in $1 \mathrm{~s}$; MRC, Medical Research Council; $\mathrm{np}$, non-parametric; $\mathrm{PaCO}_{2}$, partial pressure of carbon dioxide in arterial blood; $\mathrm{PaO}_{2}$, partial pressure of oxygen in arterial blood; SGRQ, St George's Respiratory Questionnaire.

rating scale was simple to learn and repeatable between raters. It demonstrated significant atrophy in the frontal regions of non-hypoxaemic patients with COPD with moderate airflow obstruction and health status impairment $(\mathrm{p}=0.01)$. There was no difference between the two groups in the degree of hippocampal or temporal atrophy. This population of patients with COPD had worse average cognitive function than controls but no significant correlations were found between regional atrophy and cognitive function or measures of disease severity.

The significant frontal atrophy demonstrated by visual rating in these data confirms findings from smaller singlephoton emission computerised tomography (SPECT) cerebral perfusion studies in patients with hypoxaemic COPD suggesting frontal hypoperfusion. ${ }^{1415}$ The frontal atrophy demonstrated in this study is consistent with the frontal-type cognitive decline thought to occur in patients with COPD. ${ }^{9}$

In contrast to previous studies that have demonstrated reduced hippocampal volume in patients with $\mathrm{COPD},{ }^{16}$ no significant hippocampal atrophy was found in this study. Our findings are also not concordant with recent evidence for a correlation between hippocampal volume and hypoxaemia. ${ }^{16}$

Limitations of this study may, in part, account for the lack of significant association between regional atrophy, cognitive function and disease measures. It is possible that the relatively small participant sample size hindered our ability to form important potential correlations. However the sample size is similar to other imaging studies designed to identify meaningful differences in brain pathology in a well-characterised population sample such as in this study. Age and education are known to predict cognition, while the COPD and controls were matched for age in this study, differences in educational attainment between COPD and control groups were controlled for statistically. This approach was adopted given the pragmatic considerations required to prospectively recruit education matched control subjects.

In addition, results obtained from analysing MRI scans without using volumetric measurement can only provide an estimate for the difference in atrophy between these two groups. Clear descriptions are provided in the paper in which the technique used in this study was devised. ${ }^{22}$ However, there is still the potential for misratings as a consequence of structural variation between brains and subjectivity of raters. Moreover, the scale suggested for use with this technique is relatively narrow, probably because it was originally developed for assessing different forms of neurodegeneration. This narrow scale makes it more difficult to distinguish subtle structural changes, which are more likely to be found in patients with COPD.

This study confirms that using a simple visual rating scale is effective for investigating atrophy in the brains of patients with COPD, advocating its use in future studies. The validated technique used in this study was chosen as it was inexpensive and specialist equipment was not required. The findings of this study have implications for our understanding of structural damage in the brains of patients with COPD, supporting a possible frontal atrophy and executive pattern cognitive impairment. Like many of the studies of brain pathology and cognitive function in COPD, consistent associations between demographic and disease severity are seldom identified. There are a number of possible explanations for this. First, the principle of 'cognitive reserve'- the recognition that cross-sectional studies are unable to capture cognitive decline over time, in particular those of premorbidly 'high-normal' cognition who remain cognitively intact at time of assessment but who nevertheless may have undergone significant cognitive decline. Second, our traditional measures of disease severity have historically focused on lung physiology such as $\mathrm{FEV}_{1}$. It is now appreciated that they do not reflect the multisystem nature of COPD and are not thought to be directly involved in the pathophysiology of comorbidity in COPD. Systemic inflammation, accelerated ageing, autonomic dysfunction and genetic susceptibility have all been considered as possible mechanisms of increased comorbidity such as cognitive impairment and warrant further investigation. ${ }^{32}$

This was a preliminary study that warrants replicating in a larger sample using patients with a wider range in severity of COPD. Doing so will improve our understanding of to what extent hypoxaemia plays a role in regional atrophy and also increase the likelihood of 
identifying relationships between regions of atrophy and cognitive dysfunction.

\section{CONCLUSIONS}

It is possible to use simple visual rating scale to assess regional atrophy in COPD. This technique shows evidence of frontal cerebral atrophy in patients with stable, non-hypoxaemic COPD independent of comorbidity, smoking and mood. These morphological changes may contribute to the cognitive impairment displayed in this population; however, this study did not find significant correlations between the degree of brain atrophy and cognitive functioning or disease severity. Further investigations with a larger sample size are needed to explore these important potential associations. At this stage, the mechanism behind regional atrophy in the brains of patients with COPD is unclear.

Contributors JWD and CP conceived the presented idea. JWD provided data set from previous published research for the further analysis of this study. CCS performed image analysis and generation of results with CP. PHAD provided critical feedback and all authors contributed to shaping of the research. CCS wrote the manuscript with supervision from JWD, CP and PHAD.

Funding The authors have not declared a specific grant for this research from any funding agency in the public, commercial or not-for-profit sectors.

Competing interests None declared.

Patient consent Not required.

Ethics approval Wandsworth and East Central London Research Ethics Committees. Provenance and peer review Not commissioned; externally peer reviewed. Data sharing statement № additional data are available.

Open access This is an open access article distributed in accordance with the Creative Commons Attribution Non Commercial (CC BY-NC 4.0) license, which permits others to distribute, remix, adapt, build upon this work non-commercially, and license their derivative works on different terms, provided the original work is properly cited, appropriate credit is given, any changes made indicated, and the use is non-commercial. See: http://creativecommons.org/licenses/by-nc/4.0/

\section{REFERENCES}

1. Barnes PJ, Celli BR. Systemic manifestations and comorbidities of COPD. Eur Respir J 2009;33:1165-85.

2. Soriano JB, Visick GT, Muellerova $\mathrm{H}$, et al. Patterns of comorbidities in newly diagnosed COPD and asthma in primary care. Chest 2005;128:2099-107.

3. Maclay JD, McAllister DA, Macnee W. Cardiovascular risk in chronic obstructive pulmonary disease. Respirology 2007;12:634-41.

4. Grant I, Heaton RK, McSweeny AJ, et al. Neuropsychologic findings in hypoxemic chronic obstructive pulmonary disease. Arch Intern Med 1982;142:1470-6.

5. Dodd JW, Getov SV, Jones PW. Cognitive function in COPD. Eur Respir J 2010;35:913-22.

6. Antonelli-Incalzi R, Corsonello A, Trojano L, et al. Correlation between cognitive impairment and dependence in hypoxemic COPD. J Clin Exp Neuropsychol 2008;30:141-50.

7. Incalzi RA, Corsonello A, Pedone $\mathrm{C}$, et al. Construct validity of activities of daily living scale: a clue to distinguish the disabling effects of COPD and congestive heart failure. Chest 2005;127:830-8.
8. Incalzi RA, Gemma A, Marra C, et al. Verbal memory impairment in COPD: its mechanisms and clinical relevance. Chest 1997;112:1506-13.

9. Dodd JW, Charlton RA, van den Broek MD, et al. Cognitive dysfunction in patients hospitalized with acute exacerbation of COPD. Chest 2013;144:119-27.

10. Antonelli-Incalzi R, Corsonello A, Pedone C, et al. Drawing impairment predicts mortality in severe COPD. Chest 2006;130:1687-94.

11. Ozge $C$, Ozge A, Unal O. Cognitive and functional deterioration in patients with severe COPD. Behav Neurol 2006;17:121-30.

12. Liesker JJ, Postma DS, Beukema RJ, et al. Cognitive performance in patients with COPD. Respir Med 2004;98:351-6.

13. Dodd JW, Chung AW, van den Broek MD, et al. Brain structure and function in chronic obstructive pulmonary disease: a multimodal cranial magnetic resonance imaging study. Am J Respir Crit Care Med 2012;186:240-5

14. Ortapamuk $\mathrm{H}$, Naldoken $\mathrm{S}$. Brain perfusion abnormalities in chronic obstructive pulmonary disease: comparison with cognitive impairment. Ann Nucl Med 2006;20:99-106.

15. Antonelli Incalzi R, Marra C, Giordano A, et al. Cognitive impairment in chronic obstructive pulmonary disease. J Neuro 2003;250:325-32.

16. $\mathrm{Li}$ J, Fei GH. The unique alterations of hippocampus and cognitive impairment in chronic obstructive pulmonary disease. Respir Res 2013;14:140-9.

17. Zhang $\mathrm{H}$, Wang $\mathrm{X}$, Lin J, et al. Grey and white matter abnormalities in chronic obstructive pulmonary disease: a case-control study. BMJ Open 2012;2:e000844

18. Esser RW, Stoeckel MC, Kirsten A, et al. Structural brain changes in patients with COPD. Chest 2016;149:426-34.

19. Takeuchi H, Taki Y, Sassa Y, et al. Brain structures associated with executive functions during everyday events in a non-clinical sample. Brain Struct Funct 2013;218:1017-32.

20. Tulving E, Markowitsch HJ. Episodic and declarative memory: role of the hippocampus. Hippocampus 1998;8:198-204.

21. National Institute for Health and Care Excellence Chronic obstructive pulmonary disease. Management of chronic obstructive pulmonary disease in adults in primary and secondary care. CG101. London: NICE, 2010.

22. Davies RR, Scahill VL, Graham A, et al. Development of an $\mathrm{MRI}$ rating scale for multiple brain regions: comparison with volumetrics and with voxel-based morphometry. Neuroradiology 2009;51:491-503.

23. Charlson ME, Pompei P, Ales KL, et al. A new method of classifying prognostic comorbidity in longitudinal studies: development and validation. J Chronic Dis 1987;40:373-83.

24. Wolf PA, D'Agostino RB, O'Neal MA. The Framingham Study. Stroke 1992;23:1551-5.

25. Zigmond AS, Snaith RP. The hospital anxiety and depression scale Acta Psychiatr Scand 1983;67:361-70.

26. Folstein MF, Folstein SE, McHugh PR. "Mini-mental state". A practical method for grading the cognitive state of patients for the clinician. J Psychiatr Res 1975;12:189-98.

27. Delis DC, Kaplan E, Kramer JH. Delis-Kaplan Executive Function System (D-KEFS). San Antonio, TX: The Psychological Corporation, 2001.

28. Saklofsk DH, Schoenberg MR. Wechsler Adult Intelligence Scale (All Versions). In: Kreutzer JS, DeLuca J, DeLuca J, eds. Encyclopedia of Clinical Neuropsychology. New York, NY: Springer, 2011.

29. Meyers JE, Meyers KR. Rey Complex Figure Test and recognition trial: Professional manual. Odessa, FL: Psychological Assessment Resources, 1995

30. Wechsler D. A standardized memory scale for clinical use. J Psychol 1945;19:87-95.

31. Lezak MD, Howieson DB, Loring DW. Neuropsychological Assessment. 4th edn. New York, NY: Oxford University Press, 2004.

32. Barnes PJ. Chronic obstructive pulmonary disease: effects beyond the lungs. PLoS Med 2010;7:e1000220 